\title{
Comparison of Birth Weights of Pre-Delivery Anemic and Non-Anemic Routine Iron Supplemented Pregnant Women
}

\author{
Tolga ATAKUL ${ }^{1}$
}

Aydin, Turkey

\begin{abstract}
OBJECTIVE: To investigate the effects of pre-delivery hematological parameters on birth weight and to compare their relationship with maternal age and parity in women who had taken routine iron supplementation during pregnancy

STUDY DESIGN: A retrospective study was conducted among low-risk pregnant women who were delivered at term. Pre-delivery hematological parameters and clinical information were extracted from patient files and compared with birth weights. Women with known hematological disorders, women with the previous history of intravenous iron treatment and the ones who did not regularly use iron supplementation were excluded.

RESULTS: All patients (1066) had undergone complete blood testing before delivery. Mean values for hemoglobin, hematocrit, mean corpuscular volume, red blood cell count and red cell distribution width were as follows; $10.9 \mathrm{~g} / \mathrm{dL}( \pm 1.4), 34.6 \%( \pm 4.3)$, 84.6fL $( \pm 7.8), 4.1 \mathrm{~g} / \mathrm{dL}( \pm 0.4)$, and $14.2 \%( \pm 2.5)$, respectively. When all patients are evaluated, 300 (28\%) of them were found to be anemic according to Word Health Organization criteria but not for the former ACOG cut-off, which was $9.5 \mathrm{~g} / \mathrm{dL}$. There was no significant association between pre-delivery anemia and birth weight. However, the anemic women group was found to be slightly younger than non-anemic ones according to both criteria.

CONCLUSION: Our results documented that younger age was associated with more pronounced anemia before delivery. This study did not show any association between pre-delivery anemia and low birth weight irrespective of maternal age. There is a need for further reports investigating the prognostic importance of hemoglobin reduction during pregnancy for infant birth weight in specific age groups and placental insufficiency related conditions.
\end{abstract}

Keywords: Anemia, Birth weight, Iron supplementation, Pregnancy

Gynecol Obstet Reprod Med 2020;26(1):21-24

\section{Introduction}

Anemia is the most common hematologic abnormality both in the general population and pregnant women (1). It is documented to be a major public health problem in low and middle-income regions (2). According to WHO, more than $40 \%$ of pregnant women worldwide are anemic and in more

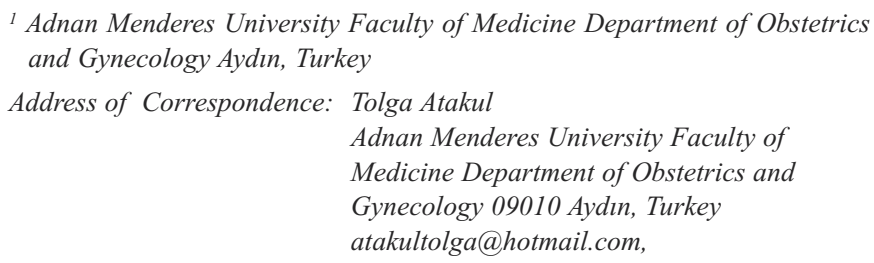

Submitted for Publication: Revised for: Publication:

20.06.2019

24.09.2019

24.02.2020

ORCID IDs of the authors:

TA: 0000-0002-0219-000X

\begin{tabular}{|c|c|}
\hline Quick Response Code: & Access this article online \\
\cline { 2 - 2 } & $\begin{array}{c}\text { Website: www.gorm.com.tr } \\
\text { e- mail: info@gorm.com.tr }\end{array}$ \\
\cline { 2 - 2 } & DOI:10.21613/GORM.2020.991 \\
\hline
\end{tabular}

How to cite this article: Atakul T. Comparison of Birth Weights of PreDelivery Anemic and Non-Anemic Routine Iron Supplemented Pregnant Women. Gynecol Obstet Reprod Med 2020;26(1):21-24 than $50 \%$ of these cases, the reason is iron deficiency (3). Notably, the most damaging consequences of anemia also occur in this population as increased maternal morbidity and mortality along with neonatal negative effects. Vitamin deficiencies (folate, vitamin B12 or A) infections, chronic diseases, and genetic diseases account for the rest of the underlying causes for anemia in pregnancy.

Pregnancy stands as a special condition for anemia because physiologic changes during pregnancy alter normal hematologic parameters. Overall, the iron requirement during the pregnancy period is increased and there is a greater expansion in plasma volume than red blood cell mass resulting in decreased hemoglobin ( $\mathrm{Hb}$ ) and hematocrit (Hct) levels (4).

The reported prevalence of anemia in pregnancy globally shows a marked variation (2). Even in the same country, it was reported that distinct differences can be observed in different ethnic populations. For example, in a report from the United States of America (USA), anemia prevalence was found to be two times higher in non-Hispanic black women than that of non-Hispanic white women (5). 
Centers for Disease Control and Prevention (CDC) acknowledges the following levels as anemic: $\mathrm{Hb}(\mathrm{g} / \mathrm{dL})$ and Hct (percentage) levels below $11 \mathrm{~g} / \mathrm{dL}$ and $33 \%$, in the first trimester; $10.5 \mathrm{~g} / \mathrm{dL}$ and $32 \%$, in the second trimester; and 11 $\mathrm{g} / \mathrm{dL}$ and $33 \%$, in the last trimester, respectively (6). According to an earlier report of ACOG however, greater ranges are suggested for the $\mathrm{Hb}$ and Hct values in the third trimester $(9.5-15.0 \mathrm{~g} / \mathrm{dL}$ for $\mathrm{Hb}$ and $28.0 \%-40.0 \%$ for $\mathrm{Hct}$ ) (1). A recent practice bulletin of ACOG differently evaluated the iron-supplemented population and considered $11 \mathrm{~g} / \mathrm{dL}$ and $33 \%$ for $\mathrm{Hb}$ and $\mathrm{Hct}$, respectively as anemic values in the third trimester. That report also stated macrocytic and microcytic anemias are associated with a mean corpuscular volume (MCV) greater than $100 \mathrm{fL}$ and lower than $80 \mathrm{fL}$, respectively (7). WHO also suggested similar cut-off values for the definition of anemia (8). WHO classified anemia in pregnancy into three grades according to its severity; mild, moderate, and severe, 10-10.9, 7-9.9, and $<7 \mathrm{~g} / \mathrm{dL}$ respectively. Interestingly, none of these reports stated an approach if there is a distinct decrease from baseline but not below the thresholds. The diagnosis of anemia besides from proposed blood test can also be confirmed by increasing hemoglobin concentrations for more than $1 \mathrm{~g} / \mathrm{dL}$ after iron treatment or in a bone marrow smear absence of bone marrow iron stores (6).

Iron supplementation during pregnancy decreases the prevalence of maternal anemia at the time of delivery and standard iron supplementation was introduced as early as 1959 (3). WHO recommends routine 30-60 mg of elemental iron supplementation during pregnancy, in which $60 \mathrm{mg}$ of the daily dose of elemental iron is preferred in anemia is a severe public health problem.

A recent Cochrane included 61 trials and evaluated the effects of routine iron supplementation during pregnancy (9). This review found that iron supplementation reduced maternal anemia by $70 \%$ at term, however, there was no difference for severe anemia in the second or third trimester. Although there was a lower frequency of low birth weight newborns and fewer preterm deliveries in women taking iron supplementation, these differences were not statistically significant. Moreover, the clinical significance of anemia at term in women taking supplementations is also without any clinical interpretation. The authors commented that routine iron supplementation in pregnancy may result in heterogeneous outcomes depending on the women's background risk and adherence to the medication. Such an analysis in the literature is lacking for the Turkish population and therefore we primarily intended to analyze the association of pre-delivery anemia severity with respect to obstetric outcomes in term deliveries having different background risk factors who took routine iron supplementation.

\section{Material and Method}

All women who were on routine pregnancy follow-up at their family physician had undergone complete blood testing before delivery at our clinic. The routine iron supplementation protocol during pregnancy follow-up was an administration of 60-100 mg of elemental iron (changing according to the formulation). Inclusion criteria for analysis are as follows; singleton pregnancy, history of routine follow-up at a family physician, taking regular iron supplementation throughout the pregnancy, taking regular folic acid supplementation during the first trimester. The following patients were excluded from the study: any known hematological disorder, pregnancy complications (preeclampsia, placental ablation, premature rupture of membranes, placental insufficiency), history of gastrointestinal surgery (except appendectomy), any course of blood transfusion due to acute obstetric bleeding. The distributions of the hematological parameters according to former ACOG criteria were calculated. The percentages of the severity of anemia were also calculated according to WHO classification. The patients were divided into anemic and non-anemic groups according to both former ACOG and WHO and data of groups were compared in terms of birth weight, maternal age, gravida, and parity.

Statistical tests were performed with PSPP 1.0.1 and R software with the EasyR plugin. Numerical variables were presented as mean \pm standard deviation (SD). Categorical parameters were presented along with their percentages. Normality tests were performed by distribution analysis and the Kolmogorov-Smirnov test. Student's $t$-test was used to compare continuous variables between groups. The chi-square and Fisher's exact tests were used for categorical variables. The level of statistical significance was accepted as $p<0.05$.

Institutional review board approval was obtained for retrospective data extraction (number of approval: 2017/1130) and the study was conducted in accordance with the Declaration of Helsinki.

\section{Results}

Pre-delivery hemoglobin levels of 1066 pregnant women delivered in a tertiary obstetric clinic were evaluated. The mean age of the patients was $30.0( \pm 5.8)$ years. Obstetric history parameters of the patients are presented in table I. Of the participants, 29 (2.7\%) were having a parity greater than four. Most of the patients were delivered via cesarean section $(\mathrm{n}=817,76.6 \%)$. The mean birth weight of the newborns was $3247( \pm 466)$ grams. All pregnancies were at term while 185 $(17.3 \%)$ of them were greater than 40 weeks of age.

Table I: Obstetric history of the patients

\begin{tabular}{ll}
\hline Obstetric History & Mean \pm SD \\
\hline Gravida & $2.35 \pm 1.38$ \\
Parity & $1.02 \pm 1.07$ \\
Abortion & $0.31 \pm 0.66$ \\
Living sibling & $0.99 \pm 1.03$ \\
\hline
\end{tabular}


All patients had undergone complete blood testing before delivery. Hemoglobin and other hematological parameters were also evaluated according to former and new ACOG references (1). Mean values for hemoglobin $(\mathrm{Hb})$, hematocrit (Hct), mean corpuscular volume (MCV), red blood cell count (RBC) and red cell distribution width (RDW) were as follows; $10.9 \mathrm{~g} / \mathrm{dL}( \pm 1.4), 34.6 \%( \pm 4.3), 84.6 \mathrm{fL}( \pm 7.8), 4.1 \mathrm{~g} / \mathrm{dL}( \pm 0.4)$, and $14.2 \%( \pm 2.5)$, respectively. According to these references, patients were divided into three groups (below normal, normal, above normal). Figure 1 represents the distribution of the hematological parameters according to former ACOG criteria. According to these criteria, only $80(7.5 \%)$ of the women were regarded as anemic. According to WHO's cut-off values, however, $380(35.6 \%)$ women were found to be anemic. Grading of the anemic patients according to WHO is presented in table II.

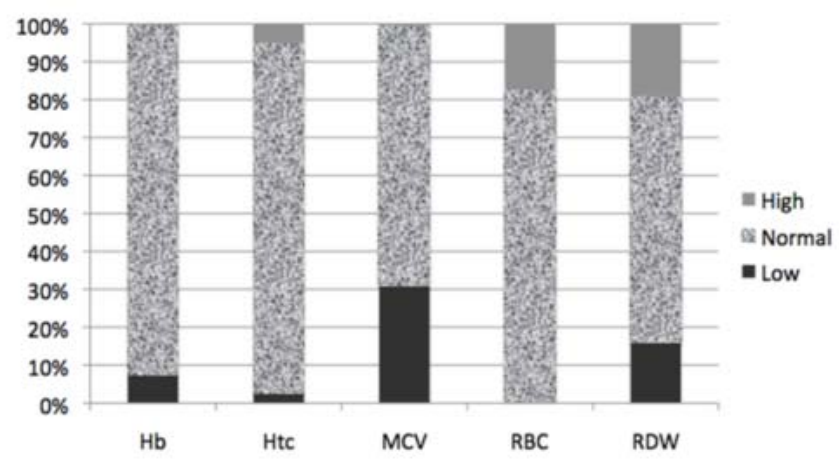

Figure 1: Distribution of the hematological parameters according to former ACOG criteria (1)

When all patients are evaluated, $300(28 \%)$ of them were found to be anemic according to WHO criteria but not for the former ACOG cut-off, which was $9.5 \mathrm{~g} / \mathrm{dL}$. Both criteria were analyzed with respect to birth weight. However, none of the comparisons yielded a significant difference (Table III).

Predelivery hematological parameters were also analyzed with respect to maternal age and parity (Table IV). Although gravida and parity were similar among anemic and normal women, anemic women according to both criteria were found to be slightly younger than non-anemic ones.

\section{Discussion}

In this study, pregnant women who had attended to regular follow-ups were analyzed with their pre-delivery hematological parameters. Even though all women were prescribed with oral iron supplementation according to a standardized protocol, there were a considerable amount of anemic cases $(7.5 \%$ according to former ACOG criteria) just before delivery. However, this anemic status that was analyzed according to two different criteria (former ACOG and WHO criteria) was not found to be related to neonatal low birth weight. Anemic women (diagnosed according to both criteria) were found to be slightly younger than normal counterparts.

A recent report from Japan reported $\mathrm{Hb}$ levels at three-time points: early ( $<16$ weeks), mid- (16-27 weeks), and late (28-36 weeks) pregnancy (10). The researchers reported that even a mild reduction in $\mathrm{Hb}$ levels from early to mid- or late pregnancy was associated with lower birth weight, placental weight, and placental ratio. Interestingly women with the intermediate or greatest reduction had a lower risk of delivering an LBW or SGA infant. On the other hand, Whittaker et al.

Table II: Grading of the anemic patients according to WHO

\begin{tabular}{llll}
\hline & Mild & Moderate & Severe \\
& $10-10.9(\mathrm{~g} / \mathrm{dL})$ & $7-9.9(\mathrm{~g} / \mathrm{dL})$ & $<7(\mathrm{~g} / \mathrm{dL})$ \\
\hline $\mathrm{n}$ & 228 & 150 & 2 \\
$\%$ (percent) & 60 & 39.5 & 0.5 \\
\hline
\end{tabular}

Table III: Comparison of birth weight with anemia status with respect to ACOG or WHO criteria

\begin{tabular}{llll}
\hline Anemia Criteria & Anemia $(n)$ & Mean birth weight & $p$ \\
\hline ACOG & Non anemic $(n=986)$ & $3251 \pm 467$ & 0.30 \\
& Anemic $(n=80)$ & $3195 \pm 443$ & 0.47 \\
WHO & Non anemic $(n=686)$ & $3239 \pm 473$ & $3261 \pm 451$ \\
& Anemic $(n=380)$ & & \\
\hline
\end{tabular}

Table IV: Predelivery hematological parameters with respect to maternal age and parity

\begin{tabular}{lcccrrr}
\hline Patients CharacteristicsCFC & \multicolumn{2}{c}{ Former ACOG criteria } & \multicolumn{3}{c}{ WHO criteria } \\
\cline { 2 - 7 } & Non anemic $\mathrm{n}=986$ & Anemic $\mathrm{n}=80$ & $p$ & Non anemic $\mathrm{n}=686$ & Anemic $\mathrm{n}=380$ & $p$ \\
\hline Maternal age (mean $\pm \mathrm{SD})$ & $30.1 \pm 5.7$ & $28.5 \pm 5.6$ & .018 & $30.3 \pm 5.6$ & $29.3 \pm 5.9$ & .006 \\
Gravida (mean $\pm \mathrm{SD}$ ) & $2.4 \pm 1.4$ & $2.2 \pm 1.2$ & .281 & $2.3 \pm 1.4$ & $2.4 \pm 1.4$ & .686 \\
Parity (mean $\pm \mathrm{SD}$ ) & $1.0 \pm 1.0$ & $1.0 \pm 1.0$ & .780 & $1.0 \pm 1.0$ & $1.1 \pm 1.0$ & .296 \\
\hline
\end{tabular}


found no association between $\mathrm{Hb}$ changes during pre-pregnancy to late pregnancy and birth weight or placental weight when they investigated serial hematologic changes (11). The inverse association of changes in $\mathrm{Hb}$ levels from early pregnancy with birth weight may be explained with plasma volume expansion. Briefly, a lower reduction in $\mathrm{Hb}$ levels may be a result of limited plasma volume during pregnancy which may also correlate with impaired fetoplacental circulation (12).

An investigation from Turkey analyzed $\mathrm{Hb}$ levels during trimesters in 329 pregnant women (13). The authors reported that women who delivered LBW infants had a smaller but insignificant $\mathrm{Hb}$ reduction during trimesters. The study population in our analysis was standardized as all women were on regular iron supplementation. The originality of our group is the fact that these women may not show the expected $\mathrm{Hb}$ reduction. On the other hand, since iron supplementation dose in our cohort is limited to prophylactic dose, we estimated that this group would, in fact, show a gradual reduction in their $\mathrm{Hb}$ levels if they were followed from the beginning of their pregnancy. However, our data do not contain such comparison and therefore we cannot assure this possibility. This is the major limitation of our study. One important strength of our study is, however, the standardized iron supplementation protocol in our group.

In this study, we analyzed women who were taking regular iron supplementation and did not find any association between pre-delivery anemic status and low birth weight. However, our results documented that younger age was associated with more pronounced anemia before delivery. This finding is coherent with the fact that plasma volume expansion related to $\mathrm{Hb}$ reduction is a favorable finding for birth weight. However, our study design is limited to draw a definitive conclusion for this hypothesis. Therefore, there is a need for further reports investigating the prognostic importance of $\mathrm{Hb}$ reduction during pregnancy for infant birth weight and also for other placental insufficiency related conditions in women taking regular iron supplementation.

Acknowledgments: None

Source of funding: None

Conflict of interest: The author declares that he has no conflict of interest

\section{References}

1. Abbassi-Ghanavati M, Greer LG, Cunningham FG.
Pregnancy and laboratory studies: a reference table for clinicians. Obstet Gynecol. 2009;114(6):1326-31

2. World Health Organization (WHO) and United Nations Children's Fund (UNICEF). Focusing on anaemia: towards an integrated approach for effective anaemia control. 2004.http://www.who.int/nutrition/publications/micronutrients/WHOandUNICEF_statement_anaemia_en.p df (accessed 20 Nov 2018).

3. WHO. Guideline: Daily iron and folic acid supplementation in pregnant women. Geneva, World Health Organization, 2012.

4. Pitkin RM. Nutritional influences during pregnancy. Med Clin North Am. 1977;61(1):3-15.

5. Adebisi OY, Strayhorn G. Anemia in pregnancy and race in the United States: blacks at risk. Fam Med. 2005;37 (9):655-62.

6. Centers for Disease Control and Prevention. Recommendations to prevent and control iron deficiency in the United States. MMWR Recomm Rep. 1998;47(RR-3):129.

7. American College of Obstetricians and Gynecologists. ACOG Practice Bulletin No. 95: anemia in pregnancy. Obstet Gynecol. 2008;112(1):201-7.

8. WHO recommendations on antenatal care for a positive pregnancy experience. World Health Organization; Luxembourg, 2016.

9. Peña-Rosas JP, De-Regil LM, Garcia-Casal MN, Dowswell T. Daily oral iron supplementation during pregnancy. Cochrane Database Syst Rev. 2015;(7): CD004736.

10. Jwa SC, Fujiwara T, Yamanobe Y, Kozuka K, Sago H. Changes in maternal hemoglobin during pregnancy and birth outcomes. BMC Pregnancy Childbirth. 2015;15:80.

11. Whittaker PG, Macphail S, Lind T. Serial hematologic changes and pregnancy outcome. Obstet Gynecol. 1996; 88(1):33-9.

12. Steer PJ. Maternal hemoglobin concentration and birth weight. Am J Clin Nutr. 2000;71(5 Suppl):1285S-7S.

13. Bakacak M, Avci F, Ercan O, Köstü B, Serin S, Kiran G, et al. The effect of maternal hemoglobin concentration on fetal birth weight according to trimesters. J Matern Fetal Neonatal Med. 2015;28(17):2106-10. 